\title{
SUBAUTOMATA OF A GENERAL FUZZY AUTOMATON
}

\section{MOHAMMAD HORRY}

Department of Mathematics

Faculty of Chamran

Technical and Vocational University (TVU)

Kerman Branch

Kerman

Iran

e-mail: mhori@tvu.ac.ir

mohhorry@yahoo.com

\begin{abstract}
In this paper, we first define the notions of a successor of the state $q$ in a maxmin general fuzzy automaton and subautomaton of a max-min general fuzzy automaton. Then define a fuzzy subsystem of a max-min general fuzzy automaton. Finally, we derive the relationships between them.
\end{abstract}

\section{Introduction and Preliminaries}

The concept of fuzzy automata was introduced by Wee in 1967 [10].

Let $\sum$ be a non-empty finite set of symbols (alternatively called characters), called the alphabet. A string (or word) over $\sum$ is any finite 2010 Mathematics Subject Classification: 18B20.

Keywords and phrases: (general) fuzzy automata, successor, subautomaton, fuzzy subsystem.

Received November 14, 2018

(C) 2019 Scientific Advances Publishers 
sequence of symbols from $\sum . \Lambda$ denotes the empty (or null) string and $\sum^{*}$ the closure of the alphabet, i.e., the set of all words over $\sum$. The length $\ell(x)$ of the word $x \in \sum^{*}$ is the number of its letters, so $\ell(\Lambda)=0$. For a nonempty set $X, \widetilde{P}(X)$ will denote the set of all fuzzy sets on $X$ and $P(X)$ will denote the set of all subsets on $X$.

A deterministic finite-state automaton is a five-tuple denoted as $A=\left(Q, \sum, f, T, s\right)$, where $Q$ is a finite set of states, $\sum$ is a finite set of input symbols, the function $f$ from $Q \times \sum$ into $Q$ is the state transition, $T$ is a subset of $Q$ of accepting states and $s \in Q$ is the initial state.

A word $x=x_{1} x_{2} \ldots x_{n} \in \sum^{*}$ is said to be accepted by $A$ if there exist states $q_{0}, q_{1}, \ldots, q_{n}$ satisfying

(1) $q_{0}=s$,

(2) $f\left(q_{i-1}, x_{i}\right)=q_{i}$ for $i=1,2, \ldots, n$,

(3) $q_{n} \in T$.

The empty word is accepted by $A$ if and only if $s \in T$.

A nondeterministic finite-state automaton is a five-tuple denoted as $A=\left(Q, \sum, f, T, s\right)$, where $Q$ is a finite set of states, $\sum$ is a finite set of input symbols, the function $f$ from $Q \times \sum$ into $P(Q)$ is the state transition, $T$ is a subset of $Q$ of accepting states and $s \in Q$ is the initial state.

A fuzzy finite-state automaton (FFA) is a six-tuple $\widetilde{F}=\left(Q, \sum, R\right.$, $Z, \delta, \omega)$, where $Q$ is a finite set of states, $\sum$ is a finite set of input symbols, $R$ is the initial state of $\widetilde{F}, Z$ is a finite set of output symbols, 
$\delta: Q \times \sum \times Q \rightarrow[0,1]$ is the fuzzy transition function which is used to map a state (current state) into another state (next state) upon an input symbol, attributing a value in the interval $[0,1]$ and $\omega: Q \rightarrow Z$ is the output function. Associated with each fuzzy transition, there is a membership value in $[0,1]$ called the weight of the transition. The transition from state $q_{i}$ (current state) to state $q_{j}$ (next state) upon input $a_{k}$ is denoted by $\delta\left(q_{i}, a_{k}, q_{j}\right)$.

We use $\delta\left(q_{i}, a_{k}, q_{j}\right)$ to refer both to a transition and its weight in the sense that whenever $\delta\left(q_{i}, a_{k}, q_{j}\right)$ is used as a value, it refers to the weight of the transition, and otherwise, specifies the transition itself. The set of all transitions of $\widetilde{F}$ will be denoted by $\Delta$. The above definition is generally accepted as a formal definition of a fuzzy finite-state automaton $[2,3,6,7,8,9]$.

In 2004, Doostfatemeh and Kremer extended the notion of fuzzy automata and introduced the notion of general fuzzy automata [1].

In this paper, by using [1] and [11], we define the notions of a successor of the state $q$ and subautomaton of a max-min general fuzzy automaton. Then define a fuzzy subsystem of a max-min general fuzzy automaton. Finally, we derive the relationships between them.

Definition 1.1 ([1]). A general fuzzy automaton (GFA) is an eighttuple machine $\widetilde{F}=\left(Q, \sum, \widetilde{R}, Z, \widetilde{\delta}, \omega, F_{1}, F_{2}\right)$, where

(i) $Q$ is a finite set of states, $Q=\left\{q_{1}, q_{2}, \ldots, q_{n}\right\}$,

(ii) $\sum$ is a finite set of input symbols, $\sum=\left\{a_{1}, a_{2}, \ldots, a_{m}\right\}$,

(iii) $\widetilde{R}$ is the set of fuzzy start states, $\widetilde{R} \in \widetilde{P}(Q)$,

(iv) $Z$ is a finite set of output symbols, $Z=\left\{b_{1}, b_{2}, \ldots, b_{k}\right\}$,

(v) $\omega: Q \rightarrow Z$ is the output function, 
(vi) $\tilde{\delta}:(Q \times[0,1]) \times \sum \times Q \rightarrow[0,1]$ is the augmented transition function,

(vii) $F_{1}:[0,1] \times[0,1] \rightarrow[0,1]$ is the membership assignment function,

(viii) $F_{2}:[0,1]^{*} \rightarrow[0,1]$ is called the multi-membership resolution function.

We note that the function $F_{1}(\mu, \delta)$ has two parameters $\mu$ and $\delta$, where $\mu$ is the membership value of a predecessor and $\delta$ is the weight of a transition. In this definition, the process that takes place upon the transition from state $q_{i}$ to $q_{j}$ on input $a_{k}$ is represented as:

$$
\mu^{t+1}\left(q_{j}\right)=\widetilde{\delta}\left(\left(q_{i}, \mu^{t}\left(q_{i}\right)\right), a_{k}, q_{j}\right)=F_{1}\left(\mu^{t}\left(q_{i}\right), \delta\left(q_{i}, a_{k}, q_{j}\right)\right) .
$$

This means that the membership value (mv) of the state $q_{j}$ at time $t+1$ is computed by function $F_{1}$ using both the membership value of $q_{i}$ at time $t$ and the weight of the transition.

The usual options for the function $F_{1}(\mu, \delta)$ are $\max \{\mu, \delta\}, \min \{\mu, \delta\}$ and $(\mu+\delta) / 2$.

The multi-membership resolution function resolves the multimembership active states and assigns a single membership value to them.

Let $Q_{a c t}\left(t_{i}\right)$ be the fuzzy set of all active states at time $t_{i}, \forall i \geq 0$. We have $Q_{a c t}\left(t_{0}\right)=\widetilde{R}$ and

$$
Q_{a c t}\left(t_{i}\right)=\left\{\left(q, \mu^{t_{i}}(q)\right): \exists q^{\prime} \in Q_{a c t}\left(t_{i-1}\right), \exists a \in \sum, \delta\left(q^{\prime}, a, q\right) \in \Delta\right\}, \forall i \geq 1 .
$$

Since $Q_{a c t}\left(t_{i}\right)$ is a fuzzy set, in order to show that a state $q$ belongs to $Q_{a c t}\left(t_{i}\right)$ and $T$ is a subset of $Q_{a c t}\left(t_{i}\right)$, we should write: $q \in$ Domain $\left(Q_{a c t}\left(t_{i}\right)\right)$ and $T \subset \operatorname{Domain}\left(Q_{a c t}\left(t_{i}\right)\right)$. 
Hereafter, we simply denote them as: $q \in Q_{a c t}\left(t_{i}\right)$ and $T \subset Q_{a c t}\left(t_{i}\right)$.

The combination of the operations of functions $F_{1}$ and $F_{2}$ on a multimembership state $q_{j}$ leads to the multi-membership resolution algorithm.

Algorithm 1.2 ([1]) (Multi-membership resolution). If there are several simultaneous transitions to the active state $q_{j}$ at time $t+1$, the following algorithm will assign a unified membership value to it:

(1) Each transition weight $\delta\left(q_{i}, a_{k}, q_{j}\right)$ together with $\mu^{t}\left(q_{i}\right)$, will be processed by the membership assignment function $F_{1}$, and will produce a membership value. Call this $v_{i}$.

$$
v_{i}=\widetilde{\delta}\left(\left(q_{i}, \mu^{t}\left(q_{i}\right)\right), a_{k}, q_{j}\right)=F_{1}\left(\mu^{t}\left(q_{i}\right), \delta\left(q_{i}, a_{k}, q_{j}\right)\right) .
$$

(2) These membership values are not necessarily equal. Hence, they need to be processed by the multi-membership resolution function $F_{2}$.

(3) The result produced by $F_{2}$ will be assigned as the instantaneous membership value of the active state $q_{j}$,

$$
\mu^{t+1}\left(q_{j}\right)=\underset{i=1}{F_{2}}\left[v_{i}\right]=\underset{i=1}{F_{2}}\left[F_{1}\left(\mu^{t}\left(q_{i}\right), \delta\left(q_{i}, a_{k}, q_{j}\right)\right)\right],
$$

where

- $n$ is the number of simultaneous transitions to the active state $q_{j}$ at time $t+1$.

- $\delta\left(q_{i}, a_{k}, q_{j}\right)$ is the weight of a transition from $q_{i}$ to $q_{j}$ upon input $a_{k}$.

- $\mu^{t}\left(q_{i}\right)$ is the membership value of $q_{i}$ at time $t$.

- $\mu^{t+1}\left(q_{j}\right)$ is the final membership value of $q_{j}$ at time $t+1$. 
Definition 1.3 ([11]). Let $\widetilde{F}=\left(Q, \sum, \widetilde{R}, Z, \omega, \widetilde{\delta}, F_{1}, F_{2}\right)$ be a general fuzzy automaton. We define max-min general fuzzy automata as $\widetilde{F}^{*}=\left(Q, \sum, \widetilde{R}, Z, \omega, \widetilde{\delta}^{*}, F_{1}, F_{2}\right)$ such that:

$$
\widetilde{\delta}^{*}: Q_{a c t} \times \sum^{*} \times Q \rightarrow[0,1],
$$

where $Q_{a c t}=\left\{Q_{a c t}\left(t_{0}\right), Q_{a c t}\left(t_{1}\right), Q_{a c t}\left(t_{2}\right), \ldots\right\}$ and for all $i \geq 0$,

$$
\widetilde{\delta}^{*}\left(\left(q, \mu^{t_{i}}(q)\right), \Lambda, p\right)= \begin{cases}1, & q=p, \\ 0, & \text { otherwise. }\end{cases}
$$

Also, if the input at time $t_{i}$ be $u_{i}$, where $u_{i} \in \sum, \forall 1 \leq i \leq n$, then

$$
\begin{aligned}
& \widetilde{\delta}^{*}\left(\left(q, \mu^{t_{i-1}}(q)\right), u_{i}, p\right)=\widetilde{\delta}\left(\left(q, \mu^{t_{i-1}}(q)\right), u_{i}, p\right), \\
& \widetilde{\delta}^{*}\left(\left(q, \mu^{t_{i-1}}(q)\right), u_{i} u_{i+1}, p\right)=\underset{q^{\prime} \in Q_{a c t}\left(t_{i}\right)}{\bigvee}\left(\widetilde { \delta } ( ( q , \mu ^ { t _ { i - 1 } } ( q ) ) , u _ { i } , q ^ { \prime } ) \wedge \widetilde { \delta } \left(\left(q^{\prime}, \mu^{t_{i}}\right.\right.\right. \\
& \left.\left.\left.\left(q^{\prime}\right)\right), u_{i+1}, p\right)\right),
\end{aligned}
$$

and recursively

$$
\begin{aligned}
& \widetilde{\delta}^{*}\left(\left(q, \mu^{t_{0}}(q)\right), u_{1} u_{2} \ldots u_{n}, p\right)=\vee\left\{\tilde { \delta } ( ( q , \mu ^ { t _ { 0 } } ( q ) ) , u _ { 1 } , p _ { 1 } ) \wedge \widetilde { \delta } \left(\left(p_{1}, \mu^{t_{1}}\left(p_{1}\right)\right),\right.\right. \\
& \left.u_{2}, p_{2}\right) \wedge \ldots \wedge \widetilde{\delta}\left(\left(p_{n-1}, \mu^{t_{n-1}}\left(p_{n-1}\right)\right), u_{n}, p\right) \mid p_{1} \in Q_{a c t}\left(t_{1}\right), p_{2} \in Q_{a c t}\left(t_{2}\right), \\
& \left.\ldots, p_{n-1} \in Q_{a c t}\left(t_{n-1}\right)\right\} .
\end{aligned}
$$

If $q \in Q_{a c t}\left(t_{i}\right)$, we should write $q$ belongs to an element of $Q_{a c t}$. Hereafter, we simply denote it as: $q \in Q_{\text {act }}$.

\section{Subautomata of a General Fuzzy Automaton}

Definition 2.1. Let $\widetilde{F}^{*}=\left(Q, \sum, \widetilde{R}, Z, \omega, \widetilde{\delta}^{*}, F_{1}, F_{2}\right)$ be a max-min general fuzzy automaton and $p, q \in Q$. Then $q$ is called a successor of $p$ if there exists $x \in \sum^{*}$ such that $\widetilde{\delta}^{*}\left(\left(p, \mu^{t}(p)\right), x, q\right)>0$. 
We denoted $S(p)$ the set of all successors of $p$. If $T \subseteq Q$, then set of all successors of $T$ denoted by $S(T)$, is defined by the set $S(T)=U$ $\{S(p): p \in T\}$.

Example 2.2. Let $\widetilde{F}^{*}=\left(Q, \sum, \widetilde{R}, Z, \omega, \widetilde{\delta}^{*}, F_{1}, F_{2}\right)$ be a max-min general fuzzy automaton, where

$$
Q=\left\{q_{0}, q_{1}, q_{2}\right\}, \sum=\{a, b\}, Q_{a c t}\left(t_{0}\right)=\widetilde{R}=\left\{\left(q_{0}, \mu^{t_{0}}\left(q_{0}\right)\right)\right\}=\left\{\left(q_{0}, 1\right)\right\},
$$

$F_{1}(\mu, \delta)=\operatorname{Min}(\mu, \delta), Z=\emptyset, \omega$ and $F_{2}$ are not applicable, $\delta\left(q_{0}, a, q_{1}\right)=0.4$, $\delta\left(q_{0}, b, q_{2}\right)=0.5, \delta\left(q_{1}, a, q_{2}\right)=0.3, \delta\left(q_{2}, a, q_{2}\right)=0.2$.

If we choose the input string $x=a a \ldots a$, then

$$
\begin{aligned}
& Q_{a c t}\left(t_{1}\right)=\left\{\left(q_{1}, \mu^{t_{1}}\left(q_{1}\right)\right)\right\}, \quad Q_{a c t}\left(t_{i}\right)=\left\{\left(q_{2}, \mu^{t_{i}}\left(q_{2}\right)\right)\right\}, \forall i \geq 2, \\
& \mu^{t_{1}}\left(q_{1}\right)=\widetilde{\delta}\left(\left(q_{0}, \mu^{t_{0}}\left(q_{0}\right)\right), a, q_{1}\right)=F_{1}\left(\mu^{t_{0}}\left(q_{0}\right), \delta\left(q_{0}, a, q_{1}\right)\right)=F_{1}(1,0.4)=0.4, \\
& \mu^{t_{2}}\left(q_{2}\right)=\widetilde{\delta}\left(\left(q_{1}, \mu^{t_{1}}\left(q_{1}\right)\right), a, q_{2}\right)=F_{1}\left(\mu^{t_{1}}\left(q_{1}\right), \delta\left(q_{1}, a, q_{2}\right)\right)=F_{1}(0.4,0.3)=0.3, \\
& \mu^{t_{3}}\left(q_{2}\right)=\widetilde{\delta}\left(\left(q_{2}, \mu^{t_{2}}\left(q_{2}\right)\right), a, q_{2}\right)=F_{1}\left(\mu^{t_{2}}\left(q_{2}\right), \delta\left(q_{2}, a, q_{2}\right)\right)=F_{1}(0.3,0.2)=0.2, \\
& \mu^{t_{4}}\left(q_{2}\right)=\widetilde{\delta}\left(\left(q_{2}, \mu^{t_{3}}\left(q_{2}\right)\right), a, q_{2}\right)=F_{1}\left(\mu^{t_{3}}\left(q_{2}\right), \delta\left(q_{2}, a, q_{2}\right)\right)=F_{1}(0.2,0.2)=0.2, \\
& \mu^{t_{i}}\left(q_{2}\right)=0.2, \forall i \geq 5, \\
& \widetilde{\delta}^{*}\left(\left(q_{0}, \mu^{t_{0}}\left(q_{0}\right)\right), \Lambda, q_{0}\right)=1 \Rightarrow q_{0} \in S\left(q_{0}\right), \\
& \widetilde{\delta}^{*}\left(\left(q_{0}, \mu^{t_{0}}\left(q_{0}\right)\right), a, q_{1}\right)=0.4 \Rightarrow q_{1} \in S\left(q_{0}\right), \\
& \widetilde{\delta}^{*}\left(\left(q_{0}, \mu^{t_{0}}\left(q_{0}\right)\right), a a, q_{2}\right)=0.4 \wedge 0.3=0.3 \Rightarrow q_{2} \in S\left(q_{0}\right), \\
& \widetilde{\delta}^{*}\left(\left(q_{1}, \mu^{t_{1}}\left(q_{1}\right)\right), a, q_{2}\right)=0.3 \Rightarrow q_{2} \in S\left(q_{1}\right) .
\end{aligned}
$$


Definition 2.3. Let $\widetilde{F}^{*}=\left(Q, \sum, \widetilde{R}, Z, \omega, \widetilde{\delta}^{*}, F_{1}, F_{2}\right)$ be a max-min general fuzzy automaton and $\widetilde{F}^{*^{\prime}}=\left(T, \sum, \widetilde{R}, Z, \omega, \widetilde{\delta}^{*^{\prime}}, F_{1}, F_{2}\right)$. Then $\widetilde{F}^{*^{\prime}}$ is called a subautomaton of $\widetilde{F}^{*}$ if $S(T) \subseteq T$, where $\widetilde{\delta}^{*^{\prime}}=\left.\widetilde{\delta}^{*}\right|_{\left(Q_{a c t} \cap T\right) \times \sum^{*} \times T}$.

Definition 2.4. Let $\widetilde{F}^{*}=\left(Q, \sum, \widetilde{R}, Z, \omega, \widetilde{\delta}^{*}, F_{1}, F_{2}\right)$ be a max-min general fuzzy automaton and $\lambda$ be a fuzzy subset on $Q$. $\lambda$ is called a fuzzy subsystem of $\widetilde{F}^{*}$, if

$$
\lambda(q) \geq \lambda(p) \wedge \widetilde{\delta}\left(\left(p, \mu^{t}(p)\right), a, q\right), \forall p, q \in Q, a \in \sum .
$$

Example 2.5. In Example 2.2, let $\lambda\left(q_{0}\right)=0.3, \lambda\left(q_{1}\right)=0.4$ and $\lambda\left(q_{2}\right)$ $\lambda\left(q_{2}\right)=0.5$. If we choose the input string $x=a a \ldots a$, then

$$
\begin{aligned}
& Q_{a c t}\left(t_{1}\right)=\left\{\left(q_{1}, \mu^{t_{1}}\left(q_{1}\right)\right)\right\}, \quad Q_{a c t}\left(t_{i}\right)=\left\{\left(q_{2}, \mu^{t_{i}}\left(q_{2}\right)\right)\right\}, \forall i \geq 2, \\
& \mu^{t_{1}}\left(q_{1}\right)=\widetilde{\delta}\left(\left(q_{0}, \mu^{t_{0}}\left(q_{0}\right)\right), a, q_{1}\right)=F_{1}\left(\mu^{t_{0}}\left(q_{0}\right), \delta\left(q_{0}, a, q_{1}\right)\right)=F_{1}(1,0.4)=0.4, \\
& \mu^{t_{2}}\left(q_{2}\right)=\widetilde{\delta}\left(\left(q_{1}, \mu^{t_{1}}\left(q_{1}\right)\right), a, q_{2}\right)=F_{1}\left(\mu^{t_{1}}\left(q_{1}\right), \delta\left(q_{1}, a, q_{2}\right)\right)=F_{1}(0.4,0.3)=0.3, \\
& \mu^{t_{3}}\left(q_{2}\right)=\widetilde{\delta}\left(\left(q_{2}, \mu^{t_{2}}\left(q_{2}\right)\right), a, q_{2}\right)=F_{1}\left(\mu^{t_{2}}\left(q_{2}\right), \delta\left(q_{2}, a, q_{2}\right)\right)=F_{1}(0.3,0.2)=0.2, \\
& \mu^{t_{4}}\left(q_{2}\right)=\widetilde{\delta}\left(\left(q_{2}, \mu^{t_{3}}\left(q_{2}\right)\right), a, q_{2}\right)=F_{1}\left(\mu^{t_{3}}\left(q_{2}\right), \delta\left(q_{2}, a, q_{2}\right)\right)=F_{1}(0.2,0.2)=0.2, \\
& \mu^{t_{i}}\left(q_{2}\right)=0.2, \forall i \geq 5, \\
& \lambda\left(q_{0}\right) \geq \lambda\left(q_{0}\right) \wedge \widetilde{\delta}^{*}\left(\left(q_{0}, \mu^{t_{0}}\left(q_{0}\right)\right), \Lambda, q_{0}\right)=0.3 \wedge 1=0.3, \\
& \lambda\left(q_{1}\right) \geq \lambda\left(q_{0}\right) \wedge \widetilde{\delta}^{*}\left(\left(q_{0}, \mu^{t_{0}}\left(q_{0}\right)\right), a, q_{1}\right)=0.3 \wedge 0.4=0.3, \\
& \lambda\left(q_{2}\right) \geq \lambda\left(q_{1}\right) \wedge \widetilde{\delta}^{*}\left(\left(q_{1}, \mu^{t_{1}}\left(q_{1}\right)\right), a, q_{2}\right)=0.4 \wedge 0.3=0.3 .
\end{aligned}
$$

Thus $\lambda$ is a fuzzy subsystem of $\widetilde{F}^{*}$. 
Theorem 2.6. Let $\widetilde{F}^{*}=\left(Q, \sum, \widetilde{R}, Z, \omega, \widetilde{\delta}^{*}, F_{1}, F_{2}\right)$ be a max-min general fuzzy automaton and $\lambda$ be a fuzzy subset on $Q$. Then $\lambda$ is a fuzzy subsystem of $\widetilde{F}^{*}$ if and only if $\lambda(q) \geq \lambda(p) \wedge \widetilde{\delta}^{*}\left(\left(p, \mu^{t}(p)\right), x, q\right), \forall p, q \in Q$, $x \in \sum^{*}$.

Proof. Let $\lambda$ be a fuzzy subsystem of $\widetilde{F}^{*}$ and $p, q \in Q, x \in \sum^{*}$. We prove the theorem by mathematical induction on $|x|=n$. If $n=0$, then $x=\Lambda . \quad$ Now $\quad$ if $\quad q=p$, then $\lambda(p) \wedge \widetilde{\delta}^{*}\left(\left(p, \mu^{t}(p)\right), x, q\right)=\lambda(p) \wedge 1=$ $\lambda(p)=\lambda(q)$. If $q \neq p$, then $\lambda(p) \wedge \widetilde{\delta}^{*}\left(\left(p, \mu^{t}(p)\right), x, q\right)=\lambda(p) \wedge 0=0$ $\leq \lambda(q)$.

Assume that the theorem is true for all $u \in \sum^{*}$ such that $|u|=n-1$, $n>1$. Let $x=a u$, where $a \in \sum$ and $|u|=n-1$. Then we have

$$
\begin{aligned}
\lambda(p) \wedge \widetilde{\delta}^{*}\left(\left(p, \mu^{t}(p)\right), x, q\right)=\lambda(p) \wedge \widetilde{\delta}^{*}\left(\left(p, \mu^{t}(p)\right), a u, q\right) \\
\quad=\lambda(p) \wedge\left(\bigvee_{r \in Q} \widetilde{\delta}^{*}\left(\left(p, \mu^{t}(p)\right), a, r\right) \wedge \widetilde{\delta}^{*}\left(\left(r, \mu^{t}(r)\right), u, q\right)\right) \\
\quad=\bigvee_{r \in Q}\left\{\left(\lambda(p) \wedge \widetilde{\delta}^{*}\left(\left(p, \mu^{t}(p)\right), a, r\right)\right) \wedge \widetilde{\delta}^{*}\left(\left(r, \mu^{t}(r)\right), u, q\right)\right\} \\
\quad \leq \bigvee_{r \in Q}\left(\lambda(r) \wedge \widetilde{\delta}^{*}\left(\left(r, \mu^{t}(r)\right), u, q\right)\right) \leq \lambda(q) .
\end{aligned}
$$

The converse is trivial.

Theorem 2.7. Let $\widetilde{F}^{*}=\left(Q, \sum, \widetilde{R}, Z, \omega, \widetilde{\delta}^{*}, F_{1}, F_{2}\right)$ be a max-min general fuzzy automaton. Then every constant fuzzy set $\lambda$ on $Q$ is a fuzzy subsystem of $\widetilde{F}^{*}$. 
Proof. Let $\lambda$ is constant fuzzy set on $Q$. So for any $p, q \in Q$, we have $\lambda(p)=\lambda(q)$. Then for any $a \in \sum$, clearly $\lambda(q)=\lambda(p) \geq \lambda(p) \wedge \widetilde{\delta}$ $\left(\left(p, \mu^{t}(p)\right), a, q\right)$. Therefore, $\lambda$ is a fuzzy subsystem of $\widetilde{F}^{*}$.

Theorem 2.8. Let $\widetilde{F}^{*}=\left(Q, \sum, \widetilde{R}, Z, \omega, \widetilde{\delta}^{*}, F_{1}, F_{2}\right)$ be a max-min general fuzzy automaton, $\lambda_{1}$ and $\lambda_{2}$ be fuzzy subsystems of $\widetilde{F}^{*}$. Then $\lambda_{1} \cap \lambda_{2}$ and $\lambda_{1} \cup \lambda_{2}$ are fuzzy subsystems of $\widetilde{F}^{*}$.

Proof. Since $\lambda_{1}$ and $\lambda_{2}$ are fuzzy subsystems of $\widetilde{F}^{*}$, for $p, q \in Q$, $x \in \sum^{*}$, we have $\lambda_{1}(q) \geq \lambda_{1}(p) \wedge \widetilde{\delta}^{*}\left(\left(p, \mu^{t}(p)\right), x, q\right)$ and $\lambda_{2}(q) \geq \lambda_{2}(p)$ $\wedge \widetilde{\delta}^{*}\left(\left(p, \mu^{t}(p)\right), x, q\right)$. So

$$
\left(\lambda_{1} \cap \lambda_{2}\right)(q)=\lambda_{1}(q) \wedge \lambda_{2}(q) \geq\left(\lambda_{1}(p) \wedge \lambda_{2}(p)\right) \wedge \widetilde{\delta}^{*}\left(\left(p, \mu^{t}(p)\right), x, q\right) .
$$

Hence, $\lambda_{1} \cap \lambda_{2}$ is a fuzzy subsystem of $\widetilde{F}^{*}$. Also, we have

$$
\left(\lambda_{1} \cup \lambda_{2}\right)(q)=\lambda_{1}(q) \vee \lambda_{2}(q) \geq\left(\lambda_{1}(p) \vee \lambda_{2}(p)\right) \wedge \widetilde{\delta}^{*}\left(\left(p, \mu^{t}(p)\right), x, q\right) .
$$

Hence, $\lambda_{1} \cup \lambda_{2}$ is a fuzzy subsystem of $\widetilde{F}^{*}$.

Theorem 2.9. Let $\widetilde{F}^{*}=\left(Q, \sum, \widetilde{R}, Z, \omega, \widetilde{\delta}^{*}, F_{1}, F_{2}\right)$ be a max-min general fuzzy automaton and $\lambda$ be a fuzzy subsystem of $\widetilde{F}^{*}$. Then $\widetilde{F}^{*^{\prime}}=\left(\operatorname{supp}(\lambda), \sum, \widetilde{R}, Z, \omega, \widetilde{\delta}^{*^{\prime}}, F_{1}, F_{2}\right)$ is a subautomaton of $\widetilde{F}^{*}$, where $\widetilde{\delta}^{*^{\prime}}=\left.\widetilde{\delta}^{*}\right|_{\left(Q_{\text {act }} \cap \operatorname{supp}(\lambda)\right) \times \sum^{*} \times \operatorname{supp}(\lambda)}$.

Proof. Let $q \in S(\operatorname{supp}(\lambda))$. Then $q \in S(p)$, for some $p \in \operatorname{supp}(\lambda)$. So $\lambda(p)>0$. Since $q \in S(p)$, then there exists $x \in \sum^{*}$ such that $\widetilde{\delta}^{*}\left(\left(p, \mu^{t}(p)\right), x, q\right)>0$. Now, since $\lambda$ is a fuzzy subsystem of $\widetilde{F}^{*}$, then we have 


$$
\lambda(q) \geq \lambda(p) \wedge \widetilde{\delta}\left(\left(p, \mu^{t}(p)\right), x, q\right)>0 .
$$

Thus $q \in \operatorname{supp}(\lambda)$. Therefore $S(\operatorname{supp}(\lambda)) \subseteq \operatorname{supp}(\lambda)$. Hence $\widetilde{F}^{*^{\prime}}$ is a subautomaton of $\widetilde{F}^{*}$.

Theorem 2.10. Let $\widetilde{F}^{*}=\left(Q, \sum, \widetilde{R}, Z, \omega, \widetilde{\delta}^{*}, F_{1}, F_{2}\right)$ be a max-min general fuzzy automaton and $\lambda$ be a fuzzy subset of $Q$. For $x \in \sum^{*}$ and $q \in Q$, define the fuzzy subset $\lambda x$ of $Q$ by

$$
(\lambda x)(q)=\bigvee_{p \in Q}\left\{\lambda(p) \wedge \widetilde{\delta}^{*}\left(\left(p, \mu^{t}(p)\right), x, q\right)\right\}
$$

Then $\lambda$ is a fuzzy subsystem of $\widetilde{F}^{*}$ if and only if $\lambda x \subseteq \lambda, \forall x \in \sum^{*}$.

Proof. Let $\lambda$ be a fuzzy subsystem of $\widetilde{F}^{*}$ and $x \in \sum^{*}, q \in Q$. Then

$$
(\lambda x)(q)=\bigvee_{p \in Q}\left\{\lambda(p) \wedge \widetilde{\delta}^{*}\left(\left(p, \mu^{t}(p)\right), x, q\right)\right\} \leq \lambda(q)
$$

Hence, $\lambda x \subseteq \lambda$.

Conversely, let $x \in \sum^{*}$ and $q \in Q$. Then for all $p \in Q$, we have $\lambda(q) \geq(\lambda x)(q)=\bigvee_{p \in Q}\left\{\lambda(p) \wedge \widetilde{\delta}^{*}\left(\left(p, \mu^{t}(p)\right), x, q\right)\right\} \geq \lambda(p) \wedge \widetilde{\delta}^{*}\left(\left(p, \mu^{t}(p)\right), x, q\right)$

Hence, $\lambda$ is a fuzzy subsystem of $\widetilde{F}^{*}$.

Theorem 2.11. Let $\widetilde{F}^{*}=\left(Q, \sum, \widetilde{R}, Z, \omega, \widetilde{\delta}^{*}, F_{1}, F_{2}\right)$ be a max-min general fuzzy automaton, $\alpha \in[0,1]$ and $p, q \in Q$. Define the fuzzy subset $q_{\alpha} \sum^{*}$ of $Q$ by

$$
\left(q_{\alpha} \sum^{*}\right)(p)=\bigvee_{x \in \Sigma^{*}}\left\{\alpha \wedge \widetilde{\delta}^{*}\left(\left(q, \mu^{t}(q)\right), x, p\right)\right\}, \forall q \in Q
$$

Then $q_{\alpha} \sum^{*}$ is a fuzzy subsystem of $\widetilde{F}^{*}$. 
Proof. Let $x^{\prime} \in \sum^{*}$, then we have

$$
\begin{aligned}
\left(q_{\alpha} \sum^{*}\right)(p) & \wedge \widetilde{\delta}^{*}\left(\left(p, \mu^{t}(p)\right), x^{\prime}, r\right) \\
& =\bigvee_{x \in \Sigma^{*}}\left\{\alpha \wedge \widetilde{\delta}^{*}\left(\left(q, \mu^{t}(q)\right), x, p\right)\right\} \wedge \widetilde{\delta}^{*}\left(\left(p, \mu^{t}(p)\right), x^{\prime}, r\right) \\
& =\bigvee_{x \in \Sigma^{*}}\left\{\alpha \wedge \widetilde{\delta}^{*}\left(\left(q, \mu^{t}(q)\right), x, p\right) \wedge \widetilde{\delta}^{*}\left(\left(p, \mu^{t}(p)\right), x^{\prime}, r\right)\right\} \\
& \leq \bigvee_{x \in \Sigma^{*}}\left\{\alpha \wedge \widetilde{\delta}^{*}\left(\left(q, \mu^{t}(q)\right), x x^{\prime}, r\right)\right\} \\
& \leq \bigvee_{u \in \Sigma^{*}}\left\{\alpha \wedge \widetilde{\delta}^{*}\left(\left(q, \mu^{t}(q)\right), u, r\right)\right\} \\
& =\left(q_{\alpha} \sum^{*}\right)(r) .
\end{aligned}
$$

Hence, $q_{\alpha} \sum^{*}$ is a fuzzy subsystem of $\widetilde{F}^{*}$.

Theorem 2.12. Let $\widetilde{F}^{*}=\left(Q, \sum, \widetilde{R}, Z, \omega, \widetilde{\delta}^{*}, F_{1}, F_{2}\right)$ be a max-min general fuzzy automaton, $\lambda$ is a fuzzy subset of $Q, \alpha \in[0,1], q \in Q$ and $\alpha \leq \lambda(q)$. Then $\lambda$ is a fuzzy subsystem of $\widetilde{F}^{*}$ if and only if $q_{\alpha} \sum^{*} \subseteq \lambda$.

Proof. Let $\lambda$ be a fuzzy subsystem of $\widetilde{F}^{*}$. Then for $p \in Q$, we have

$$
\begin{aligned}
\left(q_{\alpha} \sum^{*}\right)(p) & =\vee_{x \in \Sigma^{*}}\left\{\alpha \wedge \widetilde{\delta}^{*}\left(\left(q, \mu^{t}(q)\right), x, p\right)\right\} \\
& \leq \vee_{x \in \Sigma^{*}}\left\{\lambda(q) \wedge \widetilde{\delta}^{*}\left(\left(q, \mu^{t}(q)\right), x, p\right)\right\} \\
& \leq \vee_{x \in \Sigma^{*}} \lambda(p)=\lambda(p) .
\end{aligned}
$$

Hence, $q_{\alpha} \sum^{*} \subseteq \lambda$. 
Conversely, let $q_{\alpha} \sum^{*} \subseteq \lambda, p \in Q$ and $u \in \sum^{*}$. If $\lambda(p)=0$ or $\widetilde{\delta}^{*}\left(\left(p, \mu^{t}(p)\right), x, q\right)=0$, then $\lambda(q) \geq \lambda(p) \wedge \widetilde{\delta}^{*}\left(\left(p, \mu^{t}(p)\right), x, q\right)=0$

Suppose $\lambda(p) \neq 0$ and $\widetilde{\delta}^{*}\left(\left(p, \mu^{t}(p)\right), x, q\right) \neq 0$. Let $\alpha=\lambda(q)$ and $u \in \sum^{*}$. Thus, by the hypothesis, $q_{\alpha} \sum^{*} \subseteq \lambda$. Then we have

$$
\begin{aligned}
\lambda(p) & \geq\left(q_{\alpha} \sum^{*}\right)(p)=\vee_{x \in \Sigma^{*}}\left\{\alpha \wedge \widetilde{\delta}^{*}\left(\left(q, \mu^{t}(q)\right), x, p\right)\right\} \\
& \geq \alpha \wedge \widetilde{\delta}^{*}\left(\left(q, \mu^{t}(q)\right), u p\right) \\
& =\lambda(q) \wedge \widetilde{\delta}^{*}\left(\left(q, \mu^{t}(q)\right), u p\right) .
\end{aligned}
$$

Hence, $\lambda$ is a fuzzy subsystem of $\widetilde{F}^{*}$.

Theorem 2.13. Let $\widetilde{F}^{*}=\left(Q, \sum, \widetilde{R}, Z, \omega, \widetilde{\delta}^{*}, F_{1}, F_{2}\right)$ be a max-min general fuzzy automaton, $\alpha \in[0,1]$ and $q \in Q$. Then $\operatorname{supp}\left(q_{\alpha} \sum^{*}\right)=S(q)$.

Proof. We have

$$
\begin{aligned}
p \in S(q) & \Leftrightarrow \exists x \in \sum^{*}: \widetilde{\delta}^{*}\left(\left(q, \mu^{t}(q)\right), x p\right)>0 \\
& \Leftrightarrow \bigvee_{x \in \Sigma^{*}}\left\{\alpha \wedge \widetilde{\delta}^{*}\left(\left(q, \mu^{t}(q)\right), x, p\right)\right\}>0 \\
& \Leftrightarrow\left(q_{\alpha} \sum^{*}\right)(p)>0 \\
& \Leftrightarrow p \in \operatorname{supp}\left(q_{\alpha} \sum^{*}\right) .
\end{aligned}
$$

Thus $\operatorname{supp}\left(q_{\alpha} \sum^{*}\right)=S(q)$. 
Definition 2.14. Let $\widetilde{F}^{*}=\left(Q, \sum, \widetilde{R}, Z, \omega, \widetilde{\delta}^{*}, F_{1}, F_{2}\right)$ be a maxmin general fuzzy automaton and $\lambda$ be a fuzzy subsystem of $\widetilde{F}^{*}$. Then $\lambda$ is called cyclic if there exist $\alpha \in[0,1]$ and $q \in Q$ with $\alpha \leq \lambda(q)$ such that $\lambda=q_{\alpha} \sum^{*}$.

In this case, we call $q_{\alpha}$ a generator of $\lambda$.

Theorem 2.15. Let $\widetilde{F}^{*}=\left(Q, \sum, \widetilde{R}, Z, \omega, \widetilde{\delta}^{*}, F_{1}, F_{2}\right)$ be a max-min general fuzzy automaton, $\alpha \in[0,1], q \in Q, \alpha \leq \lambda(q)$ and $\lambda$ be a fuzzy subsystem of $\widetilde{F}^{*}$. If $\lambda$ be cyclic with generator $q_{\alpha}$, then $\lambda(q)=\alpha$.

Proof. Since $\lambda=q_{\alpha} \sum^{*}$, then we have

$$
\begin{aligned}
\lambda(q) & =\left(q_{\alpha} \sum^{*}\right)(q) \\
& =\bigvee_{x \in \Sigma^{*}}\left\{\alpha \wedge \widetilde{\delta}^{*}\left(\left(q, \mu^{t}(q)\right), x, q\right)\right\} \\
& =\alpha \wedge \bigvee_{x \in \Sigma^{*}}\left\{\widetilde{\delta}^{*}\left(\left(q, \mu^{t}(q)\right), x, q\right)\right\}=\alpha \wedge 1=\alpha .
\end{aligned}
$$

Theorem 2.16. Let $\widetilde{F}^{*}=\left(Q, \sum, \widetilde{R}, Z, \omega, \widetilde{\delta}^{*}, F_{1}, F_{2}\right)$ be a max-min general fuzzy automaton, $\alpha \in[0,1], q \in Q, \alpha \leq \lambda(q)$ and $\lambda$ be a fuzzy subsystem of $\widetilde{F}^{*}$. If $\lambda$ be cyclic with generator $q_{\alpha}$, then $\lambda(q) \geq \lambda(p)$, $\forall p \in Q$. 
Proof. Let $p \in Q$. Since $\lambda=q_{\alpha} \sum^{*}$, then we have

$$
\begin{aligned}
\lambda(p) & =\left(q_{\alpha} \sum^{*}\right)(p) \\
& =\bigvee_{x \in \Sigma^{*}}\left\{\alpha \wedge \widetilde{\delta}^{*}\left(\left(q, \mu^{t}(q)\right), x, p\right)\right\} \\
& \leq \bigvee_{x \in \Sigma^{*}}\left\{\lambda(q) \wedge \widetilde{\delta}^{*}\left(\left(q, \mu^{t}(q)\right), x, p\right)\right\} \\
& =\lambda(q) \wedge \bigvee_{x \in \Sigma^{*}}\left\{\widetilde{\delta}^{*}\left(\left(q, \mu^{t}(q)\right), x, p\right)\right\} \leq \lambda(q) .
\end{aligned}
$$

Theorem 2.17. Let $\widetilde{F}^{*}=\left(Q, \sum, \widetilde{R}, Z, \omega, \widetilde{\delta}^{*}, F_{1}, F_{2}\right)$ be a max-min general fuzzy automaton, $\alpha \in[0,1], q \in Q, \alpha \leq \lambda(q)$ and $\lambda$ be a fuzzy subsystem of $\widetilde{F}^{*}$. If $\lambda$ be cyclic with generator $q_{\alpha}$, then for any fuzzy subsystem $\lambda^{\prime}$ of $\widetilde{F}^{*}$ such that $\lambda^{\prime} \subseteq \lambda$ and $\lambda^{\prime}(q) \geq \lambda^{\prime}(r), \forall r \in Q$, we have $\lambda^{\prime}=q_{\lambda^{\prime}(q)} \sum^{*}$

Proof. Let $p \in Q$. Since $\lambda^{\prime} \subseteq \lambda$, we have $\lambda^{\prime}(p) \leq \lambda(p)$. Then $\lambda^{\prime}(p)=\lambda^{\prime}(p) \wedge \lambda(p)$. Also, since $\lambda=q_{\alpha} \sum^{*}$, then we have

$$
\begin{aligned}
\lambda(p) & =\left(q_{\alpha} \sum^{*}\right)(p) \\
& =\bigvee_{x \in \Sigma^{*}}\left\{\alpha \wedge \widetilde{\delta}^{*}\left(\left(q, \mu^{t}(q)\right), x, p\right)\right\} \\
& =\bigvee_{x \in \Sigma^{*}}\left\{\lambda(q) \wedge \widetilde{\delta}^{*}\left(\left(q, \mu^{t}(q)\right), x, p\right)\right\} \\
& =\lambda(q) \wedge \bigvee_{x \in \Sigma^{*}}\left\{\widetilde{\delta}^{*}\left(\left(q, \mu^{t}(q)\right), x, p\right)\right\} .
\end{aligned}
$$


Hence

$$
\begin{aligned}
\lambda^{\prime}(p) & =\lambda^{\prime}(p) \wedge \lambda(p) \\
& =\left(\lambda^{\prime}(p) \wedge \lambda(q)\right) \wedge \bigvee_{x \in \Sigma^{*}}\left\{\widetilde{\delta}^{*}\left(\left(q, \mu^{t}(q)\right), x, p\right)\right\}
\end{aligned}
$$

Since $\lambda^{\prime}(q) \geq \lambda^{\prime}(p)$, then we have

$$
\begin{aligned}
\lambda^{\prime}(p) & \leq\left(\lambda^{\prime}(q) \wedge \lambda(q)\right) \wedge \bigvee_{x \in \Sigma^{*}}\left\{\widetilde{\delta}^{*}\left(\left(q, \mu^{t}(q)\right), x, p\right)\right\} \\
& =\lambda^{\prime}(q) \wedge \bigvee_{x \in \Sigma^{*}}\left\{\widetilde{\delta}^{*}\left(\left(q, \mu^{t}(q)\right), x, p\right)\right\} \\
& =\bigvee_{x \in \Sigma^{*}}\left\{\lambda^{\prime}(q) \wedge \widetilde{\delta}^{*}\left(\left(q, \mu^{t}(q)\right), x, p\right)\right\}=\left(q_{\lambda^{\prime}(q)} \sum^{*}\right)(p) .
\end{aligned}
$$

Therefore $\lambda^{\prime} \subseteq q_{\lambda^{\prime}(q)} \sum^{*}$. By Theorem 2.12, we have $\lambda^{\prime}=q_{\lambda^{\prime}(q)} \sum^{*}$.

\section{Conclusion}

In this paper, we have defined the notions of a successor of the state $q$ and subautomaton of a max-min general fuzzy automaton. Then have defined a fuzzy subsystem of a max-min general fuzzy automaton. Finally, we have obtained the relationships between them.

\section{References}

[1] M. Doostfatemeh and S. C. Kremer, New directions in fuzzy automata, International Journal of Approximate Reasoning 38(2) (2005), 175-214.

DOI: https://doi.org/10.1016/j.ijar.2004.08.001

[2] J. Jin, Q. Li and Y. Li, Algebraic properties of $L$-fuzzy finite automata, Information Sciences 234 (2013), 182-202.

DOI: https://doi.org/10.1016/j.ins.2013.01.018 
[3] Y. Li and W. Pedrycz, Fuzzy finite automata and fuzzy regular expressions with membership values in lattice-ordered monoids, Fuzzy Sets and Systems 156(1) (2005), 68-92.

DOI: https://doi.org/10.1016/j.fss.2005.04.004

[4] J. N. Mordeson and D. S. Malik, Fuzzy Automata and Languages: Theory and Applications, Chapman and Hall/CRC, London/Boca Raton, FL, 2002.

[5] D. S. Malik, J. N. Mordeson and M. K. Sen, On subsystems of a fuzzy finite state machine, Fuzzy Sets and Systems 68(1) (1994), 83-92.

DOI: https://doi.org/10.1016/0165-0114(94)90274-7

[6] M. Mizumoto, J. Toyoda and K. Tanaka, Some considerations on fuzzy automata, Journal of Computer and System Sciences 3(4) (1969), 409-422.

DOI: https://doi.org/10.1016/S0022-0000(69)80029-2

[7] C. L. Giles, C. W. Omlin and K. K. Thornber, Equivalence in knowledge representation: Automata, recurrent neural networks, and dynamical fuzzy systems, Proceedings of the IEEE 87(9) (1999), 1623-1640.

DOI: https://doi.org/10.1109/5.784244

[8] C. W. Omlin, K. K. Thornber and C. L. Giles, Fuzzy finite-state automata can be deterministically encoded into recurrent neural networks, IEEE Transactions on Fuzzy Systems 6(1) (1998), 76-89.

DOI: https://doi.org/10.1109/91.660809

[9] E. S. Santos, Realizations of fuzzy languages by probabilistic, max-product, and maximin automata, Information Sciences 8(1) (1975), 39-53.

DOI: https://doi.org/10.1016/0020-0255(75)90004-3

[10] W. G. Wee, On Generalizations of Adaptive Algorithms and Application of the Fuzzy Sets Concept to Pattern Classification, Ph.D. Dissertation Purdue University, IN, 1967.

[11] M. M. Zahedi, M. Horry and Kh. Abolpor, Bifuzzy (general) topology on max-min general fuzzy automata, Advances in Fuzzy Mathematics 3(1) (2008), 51-68. 